\title{
Comparison of methods for the selection of genomic biomarkers
}

\author{
Dan Lin* \\ I-Biostat, \\ Universiteit Hasselt, \\ 3590, Diepenbeek, Belgium \\ E-mail: dan.lin@uhasselt.be \\ ${ }^{*}$ Corresponding author
}

\begin{abstract}
Abel Tilahun
Department of Biostatistics, Harvard School of Public Health, 02138, Boston, MA, USA

E-mail: atilahun@sdac.harvard.edu

\section{Jose Cortinas Abrahantes}

Scientific Assessment Support Unit, European Food Safety Authority, 43100, Parma, Italy

E-mail: jose.cortinasabrahantes@efsa.europa.eu

\section{Ziv Shkedy}

I-Biostat,

Universiteit Hasselt,

3590, Diepenbeek, Belgium

E-mail: ziv.shkedy@uhasselt.be

\section{Geert Molenberghs}

\author{
I-Biostat, \\ Universiteit Hasselt, \\ 3590, Diepenbeek, Belgium \\ and \\ 3000, Katholieke Universiteit Leuven, \\ Belgium \\ E-mail: geert.molenberghs@uhasselt.be
}




\title{
Hinrich W.H. Gohlmann, Willem Talloen and Luc Bijnens
}

Janssen Pharmaceutical Companies of Johnson and Johnson, 2340, Beerse, Belgium

E-mail: hgoehlma@its.jnj.com

E-mail: wtalloen@its.jnj.com

E-mail: lbijnens@its.jnj.com

\begin{abstract}
In recent years, a lot of attention is placed on the selection and evaluation of biomarkers in microarray experiments. Two sets of biomarkers are of importance, namely therapeutic and prognostic. The therapeutic biomarkers would give us information on the response of the genes to treatment in relation to the response of the clinical outcome to the same treatments, whereas the prognostic biomarkers enable us to predict the clinical outcome irrespective of treatments and other confounding factors. In this paper, we use different methods that allow for both linear and non-linear associations to select prognostic markers for depression, the response.
\end{abstract}

Keywords: prognostics biomarkers; microarray experiments; linear associations; non-linear associations.

Reference to this paper should be made as follows: Lin, D., Tilahun, A., Abrahantes, J.C., Shkedy, Z., Molenberghs, G., Gohlmann, H.W.H., Talloen, W. and Bijnens, L. (2013) 'Comparison of methods for the selection of genomic biomarkers', Int. J. Data Mining and Bioinformatics, Vol. 8, No. 2, pp.24-41.

Biographical notes: Dan Lin holds a PhD Degree in Biostatistics from Hasselt University, Belgium. She currently works as a researcher at Hasselt University in the area of functional genomics, statistical bioinformatics. She published on dose-response microarray analysis, biomarker selection in the microarray setting.

Abel Tilahun is PhD Harvard School of Public Health Department of Biostatistics, Centre for Biostatistics in Aids Research.

Jose Cortinas Abrahantes is Scientific Officer at European Food Safety Authority. He received the BS Degree in Mathematics (1992) from Havana University, a MSc in Biostatistics (1999) and a PhD in Biostatistics (2004) from Universiteit Hasselt. He published on surrogate markers in clinical trials, on multivariate frailty models, incomplete data methods and classification methods for multivariate multi class problems.

Ziv Shkedy is an Associate Professor for Biostatistics and Bioinformatics in Hasselt University, Belgium. He is a co-author of numerous publications applying statistical methods to infectious diseases data, non-clinical experiments in early drug development and the analysis of microarray and gene expression data. Over the last 15 years, he collaborated on many project with European Organizations (ECDC, EMCDDA) about infectious diseases project and with pharmaceutical partners about clinical. non clinical and early drug development projects. Since 2007 he serve as an Associate Editor for Biometrics. 
Geert Molenberghs is Professor of Biostatistics at Universiteit Hasselt and KU Leuven in Belgium. He received a degree in Mathematics and a $\mathrm{PhD}$ in Biostatistics. He was Editor of Applied Statistics, Biometrics, and currently of Biostatistics. He is past-president of the International Biometric Society. $\mathrm{He}$ is Director of the Interuniversity Institute for Biostatistics and statistical Bioinformatics. With Geert Verbeke, Mike Kenward, Tomasz Burzykowski, Marc Buyse, and Marc Aerts, he authored books on longitudinal and incomplete data, and on surrogate marker evaluation. He received Excellence in Continuing Education Awards of the American Statistical Association, for courses at Joint Statistical Meetings.

Hinrich W.H. Gohlmann is a Principal Scientist at Janssen Research and Development, Beerse, Belgium. He studied Biology at the Technische Hochschule Darmstadt, Germany and received his Doctoral Degree from the Centre for Molecular Biology of the University of Heidelberg, Germany. His research interests are gene expression profiling using the Affymetrix technology, data analysis, and using whole genome transcriptional data as a tool for medicinal chemistry.

Willem Talloen is a Senior Statistician at Johnson and Johnson Pharmaceutical Research and Development. He has a MSc in Biostatistics (University of Hasselt) and a PhD in Biology (University of Antwerp). Before joining Johnson and Johnson in 2005, he worked for the Belgian Public Institute of Health as a statistical consultant. Some of his work resulted in a book, two book chapters, two patents and more than 30 biological and/or statistical publications. His work on gene filtering received three presentation prices (2007 Channel Network of the International Biometrics Society, 2008 MGED and 2008 AGD) and was nominated for the Janssen Business Excellence awards 2008. His main research interests lie in the statistical analysis of high-dimensional data generated by '-omics' approaches.

Luc Bijnens holds MSc and PhD Degrees in Biology from the University of Antwerp, Belgium and a MSc in Biostatistics from the University of Hasselt, Belgium. He spent the earlier part of his career in academia at the University of Antwerp, Belgium and Kisangani, Democratic Republic of Congo, and later with Bristol Meyers Squibb and the European Organization of Research and Treatment of Cancer. He joined Johnson and Johnson in 1997. He is a Visiting Professor at the centre for Statistics of the University of Hasselt and he played a major role in the professional statistics communities in Belgium and Europe.

\section{Introduction}

The selection and evaluation of genomic biomarkers play a vital role in drug discovery and development, motivating the use of apt statistical techniques to understand the complex nature of the relationship between genes and clinical outcomes. An incorrect or sub-optimal method might lead to a great loss in terms of identifying genes that otherwise could have lead to a substantial improvement in understanding the properties of the relevant clinical outcome. Biomarkers have been classified as prognostic and/or therapeutic depending on their relationship with the clinical endpoint and their response to treatments. Therapeutic genes are those that respond to treatment and possibly aid in understanding the effect of the treatment on the clinically relevant outcome. The prognostic genes, on the other hand, are the ones that are related to the response 
irrespective of treatment and other factors. These genes enable us to learn a great deal about the biological pathways between them and the response of interest. In clinical and pre-clinical microarray experiments, objectives are not always bound to classifying subjects into different groups, such as diseased and non-diseased, based on their gene expression levels. Additional objectives, which are getting momentum in recent years, are the possibility of predicting the clinical outcome using a single gene expression level or combinations thereof. The former is called genomic biomarker, while the later is termed a joint biomarker. Several methods have been suggested to select and evaluate prognostic biomarkers. Most of these methods, however, deal with a possible linear relationship between the gene expression and the response of interest. Thus, genes that have a different form of relationship with the response will not be detected, resulting in loss of relevant information, which otherwise could have been useful. In this paper, we explore the use of various parametric and non-parametric models to select prognostic biomarkers with other type of non-linear association.

The rest of this paper is organised as follows. A motivating case study is given in Section 2 followed by methods of selecting genes/metabolites in Sections 3 and 4 where we discuss different approaches for the selection of biomarkers. The application of these approaches to the motivating case study is presented in Section 5.

\section{Motivating case study}

The data result from a study aiming at discovering putative genetic and metabolite markers in human plasma samples for depression. In total there were 100 controls and 99 depressed patients in this data set, whose Hamilton-Depression (HAMD) scores were measured. Due to the missing HAMD values in some depressed patients, there were 96 depressed patients available for gene expression. However, for 31 depressed patients who had measurements after anti-depressant treatment, after removing the missing arrays measuring gene expression, data of 19 patients were available for analysis. In addition to the gene expression, storage time duration, age, gender, and season, which are believed to strongly influence the concentration levels of certain genes, were collected for each patient.

\section{Linear associations}

Selecting genes as possible biomarkers for a particular response requires quantifying the degree of association between the response of interest and the gene expression, after correcting for treatment and other possible confounding factors. The associations between the gene expression and the response could be of linear or non-linear type. If we can possibly assume that there is a linear relationship between the gene expression and the response, after accounting for a set of confounding variables, we can use two of the widely used measures of association suggested in the surrogate marker literature, namely the adjusted association and the likelihood reduction factor. In this section, we briefly introduce the two measures within the framework of microarray data. 


\subsection{A joint model for gene expression and response}

In this section, we present a joint model for gene expression and response, which allows us to address the second question of interest, namely which gene can serve as a biomarker. Without loss of generality, lets consider a pre-clinical trial, in which measurements were made for both the gene expression and the response. Let $X_{i j}$ be the $j$ th gene expression $j=1, \ldots, m$, of the $i$ th subject, $i=1, \ldots, n$, and denote the measurement for the response of primary interest by $Y_{i}$. Let $Z_{i}$ be a design matrix containing information about a set of covariates. Finally, we denote by $\hat{\alpha}_{j}$ and $\hat{\beta}$ the ML estimates of the covariate effects for the $j$ th gene expression and the response, respectively. Following Buyse et al. (2000), we define a gene-specific joint model, in which the linear predictors of the response and the gene expression are given by

$$
\begin{aligned}
& E\left(X_{i j} \mid \boldsymbol{Z}_{i}\right)=\boldsymbol{Z}_{i} \alpha_{j}, \\
& E\left(Y_{i} \mid \boldsymbol{Z}_{i}\right)=\boldsymbol{Z}_{i} \boldsymbol{\beta} .
\end{aligned}
$$

Note that equation (1) is gene-specific and, in practice, is fitted for each gene separately, a procedure which is often termed a 'gene by gene' analysis. The parameters $\alpha_{j}$ are gene-specific effects of covariates. It is further assumed that the two outcomes are normally distributed:

$$
\left(\begin{array}{c}
X_{i j} \\
Y_{i}
\end{array}\right) \sim \mathrm{N}\left[\left(\begin{array}{c}
\boldsymbol{Z}_{i} \alpha_{j} \\
\boldsymbol{Z}_{i} \boldsymbol{\beta}
\end{array}\right), \Sigma_{j}\right],
$$

where $\Sigma_{j}$ is given by

$$
\Sigma_{j}=\left(\begin{array}{cc}
\sigma_{j j} & \sigma_{j Y} \\
\sigma_{j Y} & \sigma_{Y Y}
\end{array}\right) .
$$

In the context of the evaluation of surrogate endpoints in randomised clinical trials, Buyse and Molenberghs (1998) proposed the adjusted association as a measure for surrogacy. The adjusted association is a coefficient derived from the covariance matrix of gene-specific joint model (2):

$$
\rho_{j}=\frac{\sigma_{j Y}}{\sqrt{\sigma_{j j} \sigma_{Y Y}}} .
$$

Indeed, $\rho_{j}=1$ indicates perfect correlation, in the sense that, given the biomarker (gene expression) level, a perfect prediction of the response is possible. For the special case that the only covariate included in the model is a treatment variable, joint model (1) can be rewritten as

$$
\begin{aligned}
& E\left(X_{i j} \mid Z_{i}\right)=\mu_{j}+\alpha_{j} Z_{i}, \\
& E\left(Y_{i} \mid Z_{i}\right)=\mu_{Y}+\beta Z_{i} .
\end{aligned}
$$

Here, $\alpha_{j}$ and $\beta$ are gene-specific and the outcome treatment effects, respectively, and $\mu_{j}$ and $\mu_{Y}$ are gene-specific and the response-related intercepts, respectively. Note that $\rho_{j}$ can be equal to 1 , even if the gene is not differently expressed. This type of genes can be potential prognostic biomarkers. 


\subsection{The Information-Theoretical Approach (ITA)}

The main rationale for explicitly accommodating for the two outcomes' correlation is to allow for estimation of their association. The information-theoretical approach proposed by Alonso and Molenberghs (2007), which is elegant and computationally simple, can be considered. Let us describe the method for the case of linear models, obviously containing bivariate outcomes as a particular instance. Consider the following two linear models with $Y_{i}, X_{i j}$, and $Z_{i}$ as defined earlier:

$$
E\left(Y_{i}\right)=\boldsymbol{Z}_{i} \boldsymbol{\gamma}
$$

and

$$
E\left(Y_{i} \mid X_{i j}\right)=Z_{i} \gamma+f\left(X_{i j}\right)
$$

Here, $f\left(X_{i j}\right)$ is some flexible function of the gene expression to account for a possible linear as well as non-linear relationship between the gene expression and the response of interest. Note that model (6) relates the expected value of the response to the covariates only while (7) relates it to the potential biomarker as well. Upon fitting equations (6) and (7), the degree of association can be measured by:

$$
R_{h j}^{2}=1-\exp \left(\frac{-G^{2}}{n}\right),
$$

where $G^{2}$ denotes the likelihood ratio statistics to compare equations (6) and (7), and $n$ is the sample size. To augment $R_{h j}^{2}$ with a measure for uncertainty, Alonso and Molenberghs (2007) suggested asymptotic as well as bootstrap-based confidence intervals. Note that for continuous outcomes $R_{h j}^{2}$ and the squared adjusted association $\rho^{2}$ give identical results. This method is similar to the method proposed by Bair et al. (2006) except that they only used the conditional model in equation (7) and selected genes based on a certain threshold value of the regression coefficient, $\hat{\gamma}$, of the gene. But here we try to quantify the magnitude of association using an R-square type of measure.

\section{Alternative approaches to detect non-linear associations}

The previous two approaches enable us to select genes with linear association with the response of interest. However, there might be cases where genes are non-linearly related to the response after correcting for some factors. Thus, the need to find measures that could optimally capture this possibly non-linear relationship cannot be ignored. In the following sections, we briefly outline different methods, which might be handy in such situations as outlined by Cortinas et al. (2008).

\subsection{ITA with Penalised Smoothing Splines (PSS)}

In the information-theoretic approach, we have introduced the gene expression as a covariate in a linear fashion. However, we can incorporate a flexible function to deal with a possible non-linear relationship between the gene expression and the response. One such function is penalised smoothing spline. 
We provide a brief description of the model, as is usually encountered with longitudinal data. Let $Y_{i}$ denote the response taken from subject $i$ and $X_{i j}$ be the gene expression for the $j$ th gene on subject $i$. Then Model (7) can be expressed as: $E\left(Y_{i} \mid X_{i j}\right)=Z_{i} \beta+f\left(X_{i j}\right)$, where $\mathrm{f}(\bullet)$ is some smooth function. In our case, we focus on the truncated lines basis, which is simple in formulation and performs adequately in many circumstances (Ngo and Wand, 2004). And it can be written as:

$$
E\left(Y_{i} \mid X_{i j}\right)=Z_{i} \boldsymbol{\beta}+\sum_{q=1}^{Q} b_{q}\left(X_{i j}-\kappa_{q}\right)_{+},
$$

where $k_{1}, \ldots, k_{Q}$ are a set of distinct knots in the range of $X_{i j}, X_{+}=\max (0, X)$, and $b_{q} \sim N$ $\left(0, \sigma_{b}^{2}\right)$. The knot points are selected as equally spaced quantiles of $X_{i j}$ (Ruppert et al., 2003). A stacked version of equation (9) becomes $Y=X \beta+Z b+\varepsilon$. The correspondence between the penalised spline smoother and the optimal predictor in a mixed model framework is a key feature in fitting this model. This connection offers the opportunity of using ordinary software packages for mixed models, such as, for example, SPlus, SAS, or R. Here, we use the MIXED procedure in SAS.

Fitting penalised splines using the linear mixed model approach has some appealing advantages, such as the automatic determination of the smoothing parameter, a unified framework for inference, and the flexibility with which the models can be extended (Faes et al., 2006). Similar to the conventional information-theoretical approach, here also we can use the $R_{h j}^{2}$ to quantify the association between the gene expression and the response.

\subsection{Three other methods}

The methods to follow are based on a two stage approach. In the first stage, a joint model for expression of each gene and the response will be fitted. The second stage involves applying different methods on the residuals to the response and the gene expression. Let us introduce a set of notations that will be used in the remainder of the paper. Let $r_{i}$ and $g_{i j}$ be random residuals from model (1) denoting the response and gene expression for the $j$ th gene for subject $i$.

\subsubsection{Non-linear Correlation Coefficient (NCC)}

Let us give a concise description of the non-linear correlation coefficient measure suggested by $Q$. Wang et al. (2005). The authors have demonstrated that the mutual information carried by the rank sequences, which are obtained from the original sequences, is a good measure of non-linear correlation. They later have developed the measure as a concept called non-linear correlation coefficient.

Given two random variables $X$ and $Y$, the mutual information concept is defined as:

$$
I(X ; Y)=H(X)+H(Y)-H(X, Y),
$$

$H(X)$ is the so-called Shannon entropy of the variable $X$, which is defined as:

$$
H(X)=-\sum_{i=1}^{L} p_{i} \ln p_{i},
$$

and the joint entropy of the two variables $X$ and $Y, H(X, Y)$, is defined as 


$$
H(X ; Y)=-\sum_{i=1}^{L} \sum_{j=1}^{M} p_{i j} \ln p_{i j} .
$$

Wang et al. (2005) stated that mutual information can be thought of as a generalised correlations analogous to the linear correlation coefficient, but sensitive to any relationship, not just linear dependence. But it can be seen from the definition of the mutual information that it does not range in a definite closed interval as the correlation coefficient does, which ranges over $[-1,1]$. They have given a revised version of the mutual information, which is sensitive to the general correlation of two variables as the mutual information does, while ranging over the unit interval $[0,1]$.

Considering two discrete variables $X=\left\{x_{i}\right\}_{1 \leq i \leq N}$ and $Y=\left\{y_{i}\right\}_{1 \leq i \leq N}$, they are first sorted in ascending order and placed into $b$ ranks with first $N / b$ samples in the first rank, the second $N / b$ samples in the second rank, and so on. Second, the sample pairs, $\{x, y\}$, are placed into $a b \times b$ rank grids by comparing the sample pairs to the rank sequences of $X$ and $Y$. The revised joint entropy of the two variables $X$ and $Y$ is defined as

$$
H^{r}(X ; Y)=-\sum_{i=1}^{L} \sum_{j=1}^{M} \frac{n_{i j}}{N} \log _{b} \frac{n_{i j}}{N}
$$

where $n_{i j}$ is the number of samples distributed in the $i j$ th rank grid. Now, the non-linear correlation coefficient is defined as

$$
N C C(X ; Y)=H^{r}(X)+H^{r}(Y)-H^{r}(X, Y),
$$

where $H^{r}(X)$ is the revised entropy of the variable $X$, which is defined as

$$
H^{r}(X)=-\sum_{i=1}^{L} \frac{n_{i}}{N} \log _{b} \frac{n i}{N},
$$

Note that the number of samples distributed into each rank of $X$ and $Y$ is invariant, and the total number of sample pairs is $N$, so the non-linear correlation coefficient, i.e., equation (14) can be rewritten as

$$
N C C(X ; Y)=2+\sum_{i=1}^{b^{2}} \frac{n_{i j}}{N} \log _{b} \frac{n_{i j}}{N},
$$

The non-linear correlation coefficient not only is sensitive to the non-linear correlation of two variables, but can also describe this relationship with a number ranges from the unit interval. In the maximum correlation condition, sample sequences of the two variables are exactly the same, i.e., $x_{i}=y_{i}$

\subsubsection{Random Forests $(R F)$}

A random forest is an ensemble of many identically distributed trees generated from bootstrap samples of the original data. Each tree is constructed via a regression tree algorithm. The simplest random forest with random features is formed by selecting randomly, at each node, a small group of input variables to split on. The size of the group is fixed throughout the process of growing the forest. Each tree is grown by using the 
RTA methodology without pruning. Some features of random forest worth highlighting are:

- it is an excellent classifier, comparable in accuracy to support vector machines

- it generates an internal unbiased estimate of the generalisation error as the forest building progresses

- it computes proximities between pairs of cases that can be used in clustering, locating outliers, or by scaling, giving useful views of the data

- $\quad$ it is well known that random forests avoid overfitting and it has been demonstrated to have excellent performance in comparison to other machine learning algorithms (Breiman, 2001; Svetnik et al., 2003; Meyer et al., 2003a, 2003b).

\subsubsection{Support Vector Machine (SVM)}

Support Vector Machines (SVM) is a family of efficient and excellent learning algorithms that have been used throughout a variety of applications, such as in regression and time-series prediction applications (Drucker et al., 1997; Mäuller et al., 1997; Stitson et al., 1999; Mattera and Haykin, 1999). The pivot of the SVM algorithm is a non-linear generalisation of the so-called generalised portrait algorithm developed in the sixties by Vapnik and Lerner (1963) and Vapnik and Chervonenkis (1964). SVM can also be applied to regression problems by the introduction of an alternative loss function, which include a distance measure. SVM regression uses the e-insensitive loss function, which indicate the minimum acceptable deviates of the predicted value from the actual value. This is expressed mathematically as: $-\varepsilon \leq \omega \beta_{j}-b-\alpha_{j}$. Be reminded that $\alpha_{j}$ and $\beta_{j}$ are the disease effects from the target and surrogate tissues, respectively. Geometrically, it is a bound of size $2 \varepsilon$ around the hypothesis function and any point outside this band is considered a training error. Suppose the input data can be explained by a linear model; the goal is to find a fitting hyperplane $\left\langle\omega, \beta_{j}\right\rangle+b=0$. Formally, we need to minimise $\|\omega\|^{2} / 2$, subject to the constraints $\alpha_{j}-\left\langle\omega, \beta_{j}\right\rangle-b \leq \varepsilon$ and $\left\langle\omega, \beta_{j}\right\rangle-\alpha_{j} \leq \varepsilon$. For the non-linear problems, the data $\beta_{j}$ is projected into a high dimensional feature space $\Phi\left(\beta_{j}\right)$. The optimisation problem now becomes:

$$
\min \frac{\|\omega\|^{2}}{2}+C \cdot \sum_{j}^{M}\left(\xi_{i}+\hat{\xi}_{i}\right)
$$

subject to constraints

$$
\begin{aligned}
& \alpha_{j}-\left\langle\omega, \Phi\left(\beta_{j}\right)\right\rangle-b \leq \varepsilon+\xi_{j}, \\
& \left\langle\omega, \Phi\left(\beta_{j}\right)\right\rangle-\alpha_{j} \geq \varepsilon+\xi_{j}, \\
& \xi_{j}, \varepsilon \hat{+} \xi_{j}>0 .
\end{aligned}
$$

The constrained optimisation problem in most cases can be solved more easily in its dual formulation, which is essential for extending SVM to non-linear high dimensional feature space. We employ a standard dualisation method using Lagrange multipliers, as described in Fletcher (1989) for this problem. In this paper, we have used the polynomial and the RBF kernels. The RBF kernel, as Hsu et al. (2003) pointed out, can handle the non-linear mapping and has few parameters to be controlled ( $C$ between 0.25 and 6 , with 
step of 0.25 and $\gamma$ between 0.5 and 50 with step of 0.5 ). Similar to the case of regression trees, the association measure can be computed using the ratio between the portion of the variability not explained by the model and the total variability of the residuals from the response:

$$
R D_{S V M j}=\frac{D(r)-D S V M R\left(r \mid g_{i j}\right)}{D(r)},
$$

where $D(r)=\sum_{i}^{N}\left(r_{i}-\bar{r}\right)$ is the deviance or total variability of the residuals from the response, and $\operatorname{DSVMR}\left(r\left|g_{j}\right|\right)$ is the sum of the squares of the differences between the actual value $\left(r_{i}\right)$ and their estimated value obtained when the SVM regression model is employed.

\section{Application to the case study}

\subsection{Performance of methods}

The methods discussed in the previous sections were applied to the case study introduced in Section 2. The results are summarised in Table 1 and Figures $1-5$. The $R^{2}$ and $R^{2}-\mathrm{cv}$ reported in the table are obtained from the full data set and leave-one-out cross validation. For all the five methods, $R^{2}$ indicates the degree of linear/non-linear association between difference in gene expression and difference in HAMD score between and after the treatment, as shown in Figures 1-5. From the results in Table 1, it can be learned that there is a slightly different set of genes selected by the various methods. However, seven of the top genes selected by the information-theoretic approach with the joint model and with the penalised splines (PSS) are in common. These genes can be captured by a straight lines essentially, as observed in Figure 1. There are some other genes detected by the PSS to have non-linear relationship between the HAMD change and the gene expression change before and after treatments (see Figure 3). The RF and SVM have selected different sets of genes, mostly with non-linear trends (see Figures 2 and 4), although for these genes the $R^{2}$ with leave-one-out cross validation are less than 0.5 . It seems that this data set lacks strong evidence for genes with sensible non-linear relationship.

The Non-linear Correlation Coefficient (NCC) of Wang et al. (2005) has given, for a number of genes, similar estimated values for the association measure. This might be due to the fact that this method works on the ranks of the genes rather than on the actual values. It does not conform to a linear/non-linear relationship for the top four genes, shown in Figure 5.

From the detected genes with high $R^{2}$, they not only indicate strong associations with the HAMD score, but also can be used for the prediction of the response. Based on the models used above, the predicted values of HAMD difference before and after treatment can be calculated given patients' gene expression before and after the treatment. Therefore, once these prognostic biomarker genes identified, they can be used as good predictors for prognosis of the depression (i.e., HAMD score after the treatment) based on the patient's gene expression values before and after treatment and HAMD score before treatment. 
Table 1 Results for top 20 genes using the ITA of the joint model in 1, RF, PSS, SVM, and NCC. The top seven genes from the ITA-LM (indicated by 1:7 in the table) are discovered by the PSS as top genes with high association as well. On the other hand, the RF, SVM and NCC found some other genes to have high association between the HAMD difference and the gene expression difference before and after the treatments. Gene ${ }^{a}$ is found by the PSS, RF, and SVM. The R2 reported below by using the four methods are obtained as well as $R^{2}$ with leave-one-out cross validation, except for the NCC from Wang et al. (2005)

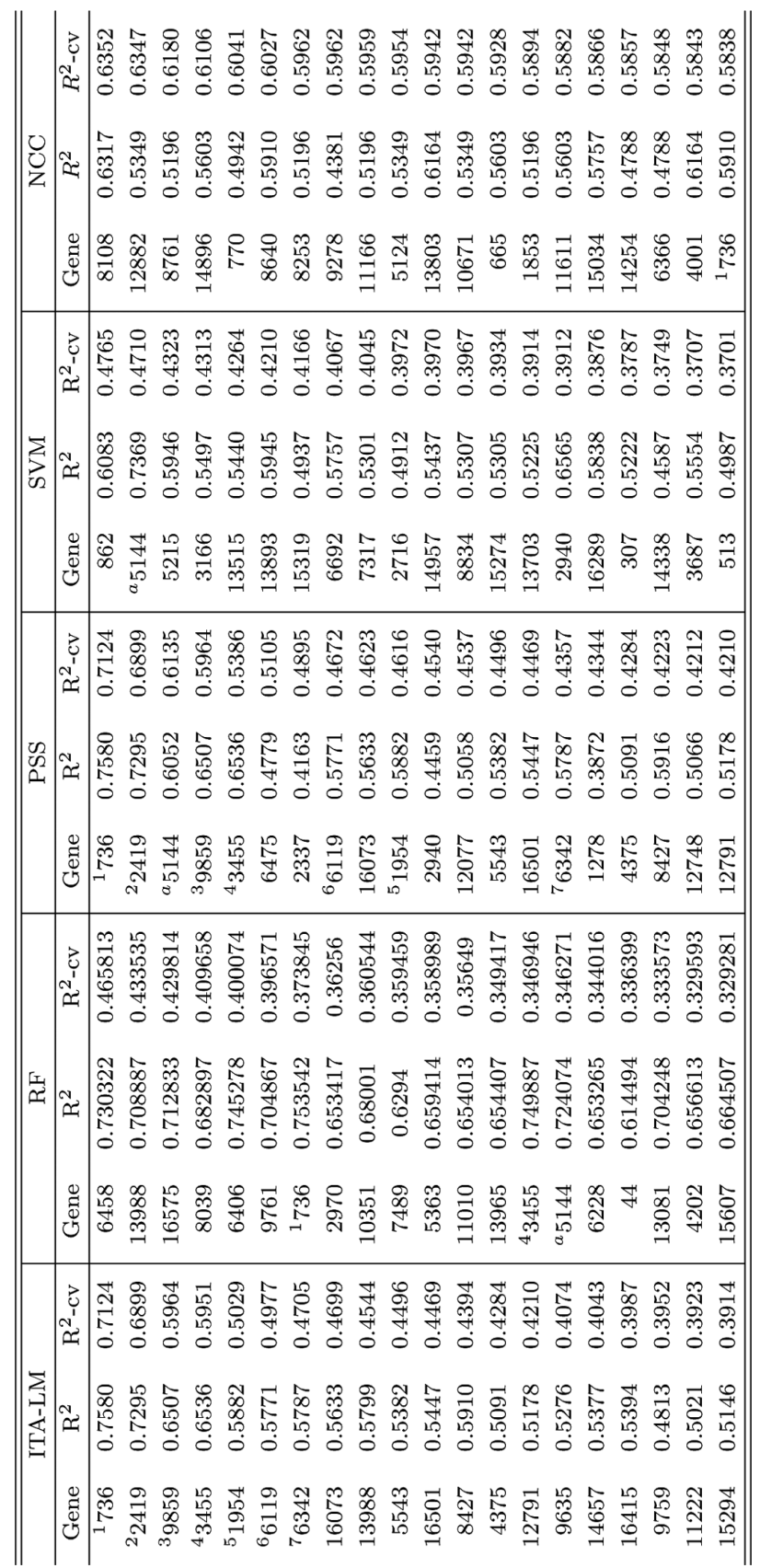


Figure 1 Scatter plot of the difference in gene expression and difference in HAMD scores before and after the treatment for top four genes found by the ITA-LM and PSS. The fitted values for the LM and PSS are the overlapping as the straight line; while the $R^{2}$ from the RF and SVM is rather low
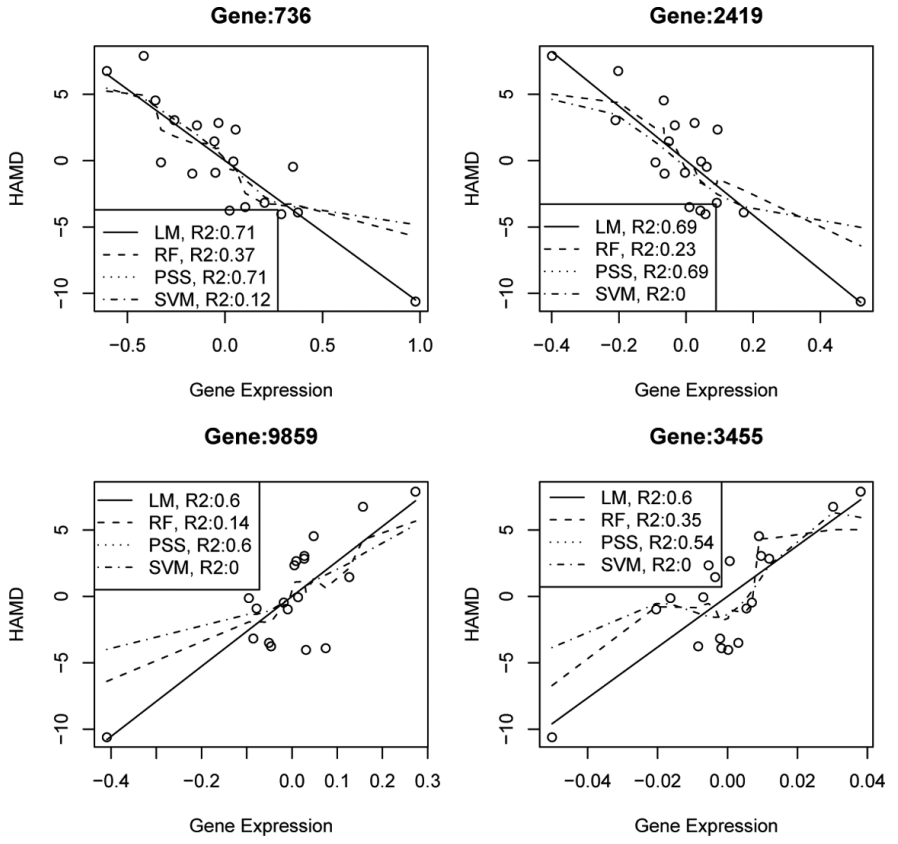

Figure 2 Scatter plot of the difference in gene expression and difference in HAMD scores before and after the treatment for four genes found by the RF

Gene:6458

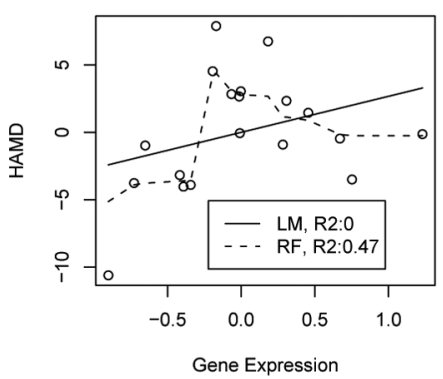

Gene:16575

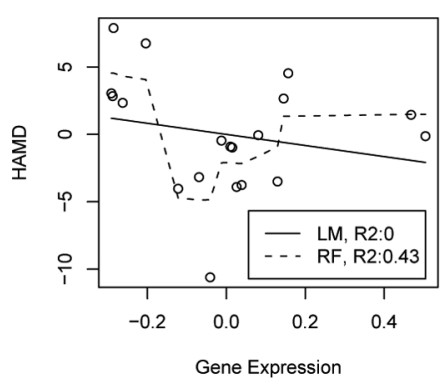

Gene:13988

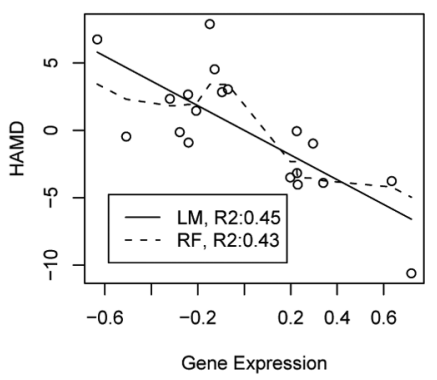

Gene:8039

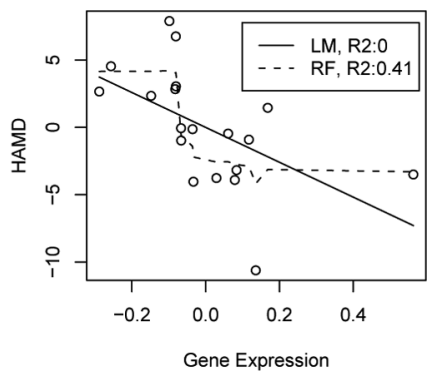


Figure 3 Scatter plot of the difference in gene expression and difference in HAMD scores before and after the treatment for four genes found by the PSS
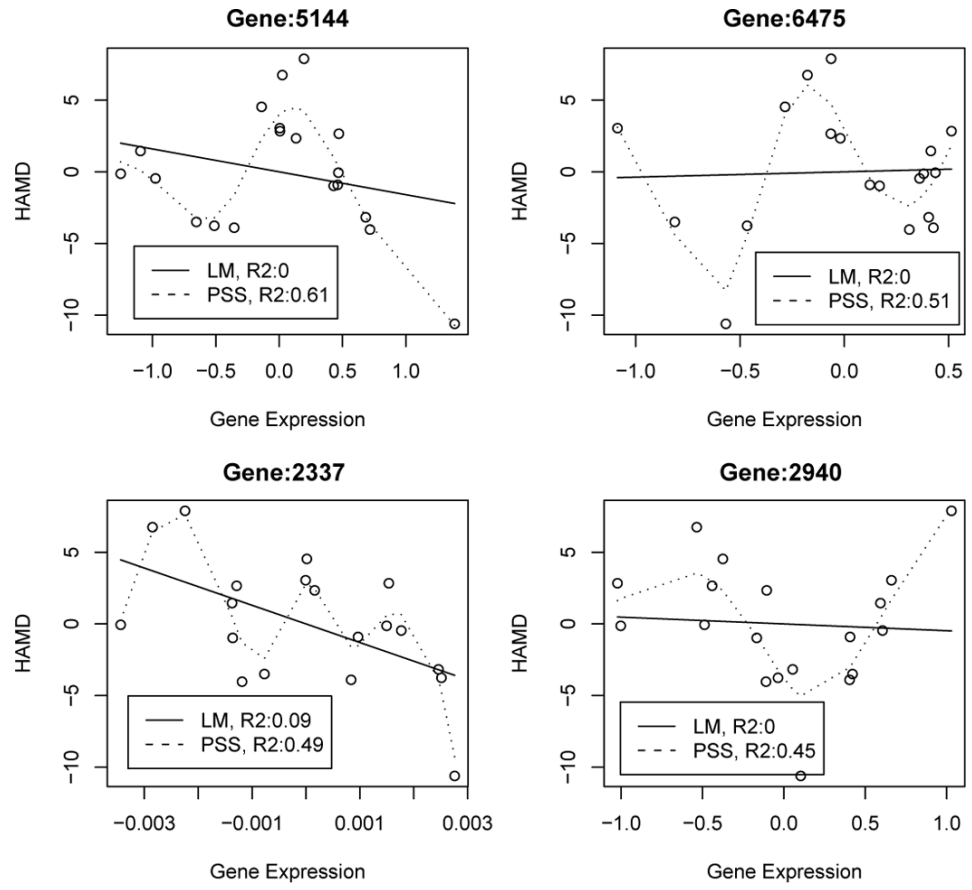

Figure 4 Scatter plot of the difference in gene expression and difference in HAMD scores before and after the treatment for four genes found by the SVM

Gene:862

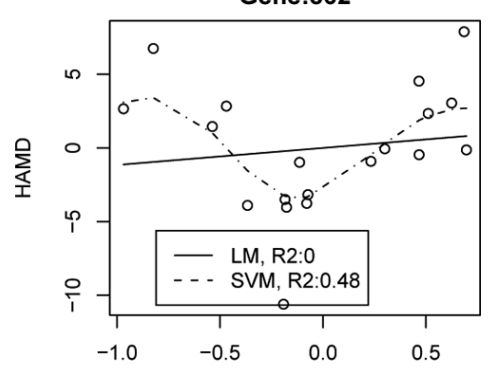

Gene Expression

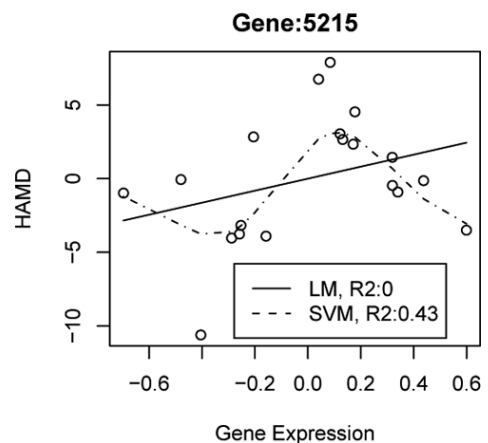

Gene:5144

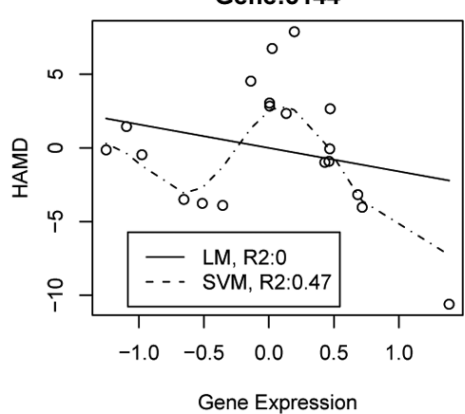

Gene:3166

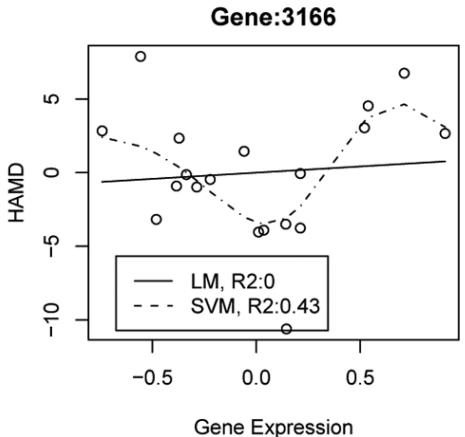


Figure 5 Scatter plot of the difference in gene expression and difference in HAMD scores before and after the treatment for four genes found by the NCC
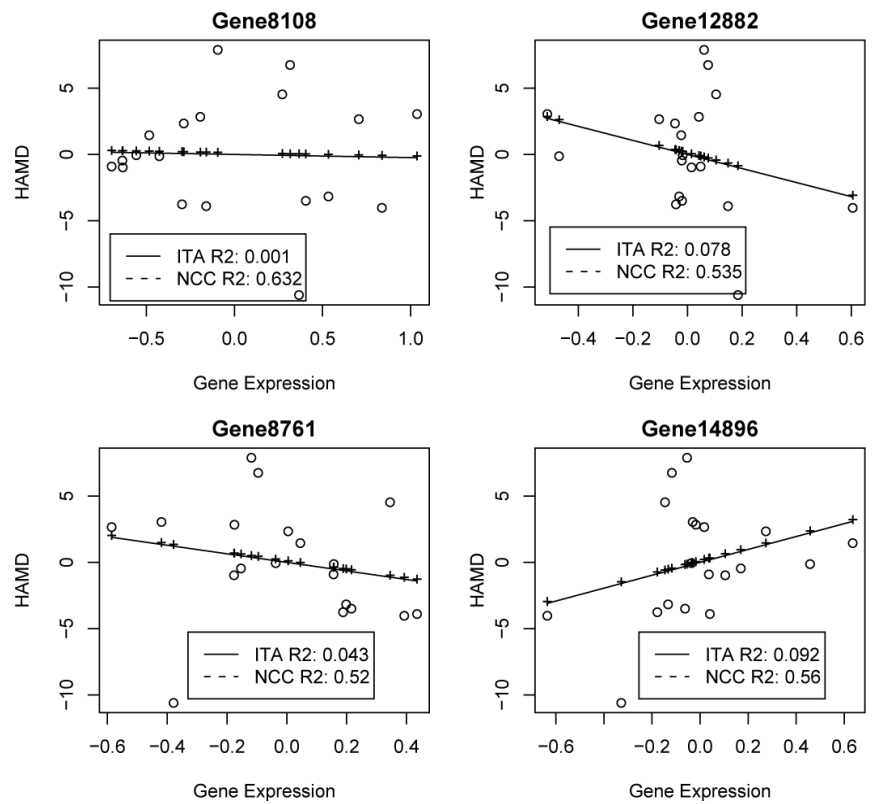

\subsection{Comparisons of methods}

Using these different methods results in different sets of genes. We use the first 100 genes from each methods to compare the agreement between methods. In particular, we use the kappa statistic to determine the degree of agreement. The overall level of agreement is captured using the kappa statistic $k$. Precisely, we compute the naive $k$, which compares the agreements to that expected if the methods were independent (Cohen, 1960). We also use the corrected measure corrected kappa statistic based on the procedure proposed by Gwet (2002) with correction to the agreement propensity for chance agreement.

The pair-wise agreements for the five methods are listed in Table 2. As we can observe from the table, that the agreement between the Non-linear Correlation Coefficient (NCC) of Wang et al. (2005) has very low agreement with all other methods applied. The highest agreement $(0.626)$ is found between the linear model and the penalised splines, followed by 0.353 between the RF and SVM approaches.

Moreover, we use the kappa's statistic to measure the agreement between more than three methods (not presented here). The highest agreement is found between the linear model, random Forest and penalised splines, which is as expected by observing the results from the pair-wise measures of agreement.

Due to the small sample size (19 patients), the reliability and reproducibility of genes found above need to be examined further. Therefore, we suggest to cross examine the list of the detected genes found by five different methods, to select those appeared in common and further study their biological functions and relationship with depression. In our case study, further research is needed to gain more biological insights of genes found in Table 1 (gene 1 to 7 and gene a). For future larger experiment, some 
cross-validation method should be implemented in an extensive manner and some resampling-based approach can be carried out to evaluate the significance of genes in order to find reliable and consistent results in genes across different methods.

Table 2 Pair-wise agreements between the five methods: corrected kappa statistics

\begin{tabular}{|c|c|c|c|c|c|}
\hline & $L M$ & $R F$ & $S P$ & $S V M$ & $N C C$ \\
\hline LM & & 0.17 & 0.626 & 0.08 & 0.049 \\
\hline $\mathrm{RF}$ & & & 0.211 & 0.353 & 0.08 \\
\hline SP & & & & 0.211 & 0.059 \\
\hline SVM & & & & & 0.069 \\
\hline
\end{tabular}

Source: Gwet (2002)

\section{Discussion}

In this paper, we have outlined a set of methods for the selection of prognostic biomarkers with the objective of identifying genes, which could have either a linear or a non-linear association with the response of interest. Methods that assume linearity might come short of identifying genes that exhibit other forms of associations resulting in loss of important prognostic biomarkers. The comparison of the methods has revealed that the methods might select different set of genes as potential biomarkers. Nevertheless, the majority of the top genes selected by the information-theoretic approach from the joint model and the penalised splines are in common. The penalised splines, RF, and SVM have detected some other genes with sensible non-linear models, but less evidence for a strong association in this data set is then found. This can be due to the small sample size in this case study, i.e., 19 subjects in total. The cross-validation procedure lacks power or is influenced by some outlying samples to obtain a strong association measure.

On the other hand, the non-linear correlation coefficient of Wang et al. (2005) performed poorly, which is reflected by the type of genes it identified as having association with the response. Moreover, this method has resulted in similar measures of association for a large number of genes, which could be attributed to the way the measure is formulated. The method uses the rankings of the measurements rather than the actual measured values for deriving an association measure. Thus, genes with similar rankings might have equal measure of association, although they might not have equal levels of association with the response.

Note that outlying observations might distort the relationship between the response and the gene expression. In some cases, a reasonably linear relationship appears to be non-linear due to a few number of outlying observations. Therefore, it is worthwhile to thoroughly investigate the type of genes that have been selected by either of the methods before making a decision to promote a gene as a possible biomarker. The use of cross-validation, although time consuming, is an important and useful practice, because it appears that the results with and without cross-validation could be substantially different. It is well known that results from the evaluation of the fit could be overly optimistic.

Based on the results here, and theoretical foundations, we can argue that the penalised splines with appropriate knot points can handle both linear as well as non-linear associations adequately. It can easily be fitted with any software which has the facility to 
handle linear mixed models and also takes substantially less computation time. Thus, it could be a prime candidate to deal with situations that call for both linear and non-linear associations. One drawback of this method is that some parametric assumptions are needed.

Finally, the methods suggested in this paper should be complemented with tests of significance for the resulting association measures through bootstrap methods, for example. Such bootstrap methods might be time consuming, but provides an important tool to evaluate the validity of some potential genes as biomarkers. In order to use these potential biomarker genes for prognosis of depression, further research is also needed in term of careful biological inference.

\section{References}

Alonso, A. and Molenberghs, G. (2007) 'Surrogate marker evaluation from an information theory perspective', Biometrics, Vol. 63, pp.180-186.

Bair, E. et al. (2006) 'Prediction by supervised principal components', J. Am. Stat. Assoc., Vol. 101, pp.119-137.

Breiman, L. (2001) 'Random forests', Machine Learning, Vol. 45, pp.5-32.

Buyse, M. and Molenberghs, G. (1998) 'The validation of surrogate endpoints in randomized experiments’, Biometrics, Vol. 54, pp.1014-1029.

Buyse, M., Molenberghs, G., Burzykowski, T., Renard, D. and Geys, H. (2000) 'The validation of surrogate endpoints in meta-analyses of randomized experiments', Biostatistics, Vol. 1, pp.49-67.

Cohen, J. (1960) 'A coefficient of agreement for nominal scales', Educ. Psychol. Meas., Vol. 20, pp.37-46.

Cortinas, J., Shkedy, Z. and Molenberghs, G. (2008) 'Alternative methods to evaluate trial level surrogacy', Clinical Trials, Vol. 5, pp.194-208.

Drucker, H., Burges, C.J.C., Kaufman, L., Smola, A. and Vapnik, V. (1997) 'Support vector regression machines', in Mozer, M.C., Jordan, M.I. and Petsche, T. (Eds.): Advances in Neural Information Processing Systems, Vol. 9, MIT Press, Cambridge, MA, pp.155-161.

Faes, C., Geys, H., Molenberghs, G., Aerts, M., Cadarso-Suarez, C., Acuna, C. and Cano, M. (2006) 'A flexible method to measure synchrony in neuronal firing', Journal of American Statistical Association, Vol. 101.

Fletcher, R. (1989) Practical Methods of Optimization, John Wiley and Sons, New York.

Gwet, K. (2002) 'Kappa statistic is not satisfactory for assessing the extent of agreement between raters', Stat. Methods Inter-Rater Reliability Assess, Vol. 1, pp.1-16.

Hsu, C.W., Chang, C.C. and Lin, C.J. (2003) A Practical Guide to Support Vector Classification, Working Paper.

Mattera, D. and Haykin, S. (1999) 'Support vector machines for dynamic reconstruction of a chaotic system', in Schölkopf, B., Burges, C.J.C. and Smola, A.J. (Eds.): Advances in Kernel Methods - Support Vector Learning, MIT Press, Cambridge, MA, pp.211-242.

Meyer, D., Leisch, F. and Hornik, K. (2003a) 'The support vector machine under test', Neurocom Putting, Vol. 55, pp.169-186.

Meyer, D., Leisch, F. and Hornik, K. (2003b) 'The support vector machine under test', Neurocomputing, Vol. 55, pp.169-186.

Ngo, L. and Wand, M.P. (2004) 'Smoothing with Mixed Model Software', Journal of Statistical Software, Vol. 9, No. 1.

Ruppert, D., Wand, M.P. and Carroll, R.J. (2003) Semiparametric Regression, Cambridge University Press, New York. 
Stitson, M., Gammerman, A., Vapnik, V., Vovk, V., Watkins, C. and Weston, J. (1999) 'Support vector regression with ANOVA decomposition kernels', in Scholkopf, B., Burges, C.J.C. and Smola, A.J. (Eds.): Advances in Kernel Methods - Support Vector Learning, MIT Press, Cambridge, MA, pp.285-292.

Svetnik, V., Liaw, A., Tong, C., Culberson, J.C., Sheridan, R.P. and Feuston, B.P. (2003) 'Random forest: a classification and regression tool for compound classification and QSAR modeling', J. Chem. Inform. Comput. Sci., Vol. 43, pp.1947-1958.

Vapnik, V. and Chervonenkis, A. (1964) 'A note on one class of perceptrons', Automation and Remote Control, p.25.

Vapnik, V. and Lerner, A. (1963) 'Pattern recognition using generalized portrait method', Automation and Remote Control, Vol. 24, pp.774-780.

Wang, Q., Shen, Y. and Zhang, J.Q. (2005) 'A nonlinear correlation measure for multivariate data set’, Physica D, Vol. 200, pp.287-295.

\section{Bibliography}

Breiman, L. (1996a) 'Bagging predictors', Machine Learning, Vol. 26, pp.123-140.

Breiman, L. (1996b) 'Heuristics of instability and stabilization in model selection', Annals of Statistics, Vol. 24, pp.2350-2383.

Breiman, L., Friedman, J.H., Olshen, R.A. and Stone, C.J. (1984) Classification and Regression Trees, Chapman \& and Hall/CRC, New York.

Burzykowski, T., Molenberghs, G. and Buyse, M. (2005) The Evaluation of Surrogate End-Points, Springer, New York.

Ding, C.G. (1996) 'On the computation of the distribution of the square of the sample multiple correlation coefficient', Computational Statistics and Data Analysis, Vol. 22, pp.345-350.

Efron, B. and Tibshirani, R. (1997) 'Improvements on cross-validation: The .632+ bootstrap method', Journal of the American Statistical Association, Vol. 92, pp.548-560.

Ihaka, R. and Gentleman, R. (1996) 'R: A Language for data analysis and graphics', Journal of Computional and Graphical Statistics, Vol. 5, pp.299-314.

Kraskov, A., Stogbauer, H. and Grassberger, P. (2004) Estimating Mutual Information, Physical Review E 69, 066138.

Lendasse, A., Wertz, V. and Verleysen, M. (2003) ICANN 2003, Joint International Conference on Artificial Neural Networks, June 26-29, 2003, Istanbul (Turkey). Artificial Neural Networks and Neural Information Processing - ICANN/ICONIP 2003, O. Kaynak, E. Alpaydin, E. Oja, L. Xu (Eds.): Springer-Verlag, Lecture Notes in Computer Science 2714, pp.573-580.

Liaw, A. and Wiener, M. (2002) Classification and regression by random forest, The Newsletter of the R Project, Vols. 2/3, pp.18-22.

Meyer, D. (2001) 'Support vector machines, the interface to libsvm in package e1071', The Newsletter of the R Project, Vols. 1/3, pp.23-26.

Meyer, D., Leisch, F. and Hornik, K. (2002) Benchmarking Support Vector Machines, Technical Report 78, SFB Adaptive Information Systems and Modeling in Economics and Management Science, p.29.

Meyer, D., Leisch, F. and Hornik, K. (2002) Benchmarking Support Vector Machines, Technical Report 78, SFB 'Adaptive information systems and modeling in economics and management science'.

Moore, D.E., Lees, B.G. and Davey, S.M. (1991) 'A new method for predicting vegetation distributions using decision tree analysis in a geographic information system', Journal of Environmental Management, Vol. 15, pp.59-71. 
Muller, K.R., Smola, A., Ratsch, G., Scholkopf, B., Kohlmorgen, J. and Vapnik V. (1997) Predicting time series with support vector machines. In: Gerstner, W., Germond, A., Hasler, M. and Nicoud J-D. (Eds.): Artificial neural networks ICANN'97, Berlin. Springer Lecture notes in computer science 1327, pp.999-1004.

Prentice, R.L. (1989) 'Surrogate endpoints in clinical trials: definitions and operational criteria', Statistics in Medicine, Vol. 8, pp.431-440.

Therneau, T.M. and Atkinson, E.J. (1997) An Introduction to Recursive Partitioning Using the Rpart Routines, Technical Report 61, Department of Health Science Research, Mayo Clinic, Rochester, New York.

Vapnik, V. (1995) The Nature of Statistical Learning Theory, Springer, New York.

Verbeke, G. and Molenberghs, G. (2000) Linear Mixed Models for Longitudinal Data, Springer, New York.

Verbyla, A.P., Cullis, B.R., Kenward, M.G. and Welham, S.J. (1999) 'The analysis of designed experiments and longitudinal data by using smoothing splines', Applied Statistics, Vol. 48, pp.269-311.

Verbyla, D.L. (1987) 'Classification trees: a new discrimination tool', Canadian Journal of Forestry Research, Vol. 17, pp.1150-1152. 